\title{
Модель термополевой нестабильности $p$-МОП-транзисторов при отрицательном смещении затвора
}

\author{
(C) О.В. Александров \\ Санкт-Петербургский государственный электротехнический университет „ЛЭТИ“, \\ 197376 Санкт-Петербург, Россия \\ E-mail: Aleksandr_ov@mail.ru
}

Поступила в Редакцию 21 марта 2019 г.

В окончательной редакции 4 сентября 2019 г.

Принята к публикации 16 сентября 2019 г.

\begin{abstract}
Разработана новая количественная модель термополевой нестабильности $p$-МОП-транзисторов при отрицательном смещении затвора. В основе модели лежит реакция депассивации поверхностных состояний на межфазной границе $\mathrm{Si}-\mathrm{SiO}_{2}$ и водородосодержащих дырочных ловушек вблизи межфазной границы $\mathrm{Si}-\mathrm{SiO}{ }_{2}$ положительно заряженными ионами водорода $\mathrm{H}^{+}$, накапливающимися в $p^{+}$-инверсном слое кремниевой подложки. Зависимости поверхностного и объемного зарядов в $p$-МОП-транзисторах от времени отрицательной термополевой нестабильности определяются кинетикой диффузии и дрейфа ионов $\mathrm{H}^{+}$из кремниевой подложки к межфазной границе $\mathrm{Si}-\mathrm{SiO}_{2}$. Влияние напряжения затвора на отрицательную термополевую нестабильность объясняется посредством влияния напряженности электрического поля на коэффициент сегрегации ионов $\mathrm{H}^{+}$на межфазной границе $\mathrm{Si}-\mathrm{SiO}_{2}$. Релаксация положительного объемного заряда, введенного в подзатворный диэлектрик при отрицательной термополевой нестабильности, описывается туннельной разрядкой оксидных ловушек электронами кремниевой подложки.
\end{abstract}

Ключевые слова: МОП-транзистор, термополевая нестабильность, поверхностные состояния, оксидные ловушки, моделирование.

DOI: 10.21883/FTP.2020.02.48901.9111

\section{1. Введение}

При отрицательном смещении затвора и повышенных температурах в кремниевых $p$-канальных МОП-транзисторах наблюдается сдвиг порогового напряжения и уменьшение подвижности носителей в канале вследствие накопления положительного заряда на поверхностных состояниях (ПС) и в подзатворном диэлектрике. Явление получило название отрицательной термополевой нестабильности (ОТПН) (negative bias temperature instability (NBTI)). ОТПН во многом определяет срок службы современных КМОП ИМС и поэтому ее исследованию уделяется повышенное внимание (см. обзоры [1-5]). ОТПН наблюдается уже при относительно невысоких электрических полях в подзатворном диэлектрике $(E=2-6 \mathrm{MB} / \mathrm{cm})$, когда нет ударной ионизации в объеме диэлектрика или в кремниевой подложке и практически отсутствует туннельная инжекция носителей из затвора. Установлено, что ОТПН является функцией времени, напряженности электрического поля и температуры. Зависимость сдвига порогового напряжения от времени описывается степенной зависимостью $\Delta V_{t} \sim t^{n}$, где показатель $n$ изменяется от $n=0.5-1$ при малых временах $\left(t=10^{-3}-10^{-2} \mathrm{c}\right)$ до $n=0.1-0.16$ при больших временах $\left(t=10^{5}-10^{6} \mathrm{c}\right)$. Зависимость от напряженности электрического поля - степенная с показателем $m=1.5-3$ или экспоненциальная. Температурная зависимость в диапазоне $100-250^{\circ} \mathrm{C}$ характеризуется энергией активации $0.1-0.3$ эВ.
Для объяснения физического механизма ОТПН была предложена реакционно-диффузионная (Р-Д) модель $[6,7]$, в основе которой лежит реакция диссоциации

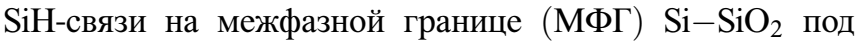
действием электрического поля:

$$
\mathrm{SiH} \leftrightarrow \mathrm{Si} \bullet+\mathrm{H},
$$

где $\mathrm{Si} \bullet$ означает оборванную связь атома кремния на $\mathrm{MФГ} \mathrm{Si}-\mathrm{SiO}_{2}\left(P_{b}\right.$-центр или поверхностное состояние). На базе Р-Д модели [6,7] был разработан ряд модифицированных моделей $[1-5,8,9]$, описывающих основные закономерности и особенности ОТПН. Учитывалось влияние дырок в реакции (1), образование водорода в различных формах - атомной $(\mathrm{H})$, молекулярной $\left(\mathrm{H}_{2}\right)$ и ионизованной $\left(\mathrm{H}^{+}\right)$, их диффузия в $\mathrm{SiO}_{2}$. Однако значения отдельных параметров в количественных Р-Д моделях $[8,9]$, в частности коэффициенты диффузии водорода в $\mathrm{SiO}_{2}$, значительно (на 5-7 порядков) ниже установленных экспериментальных значений $[10,11]$. Зависимость ОТПН от напряжения на затворе в Р-Д моделях [1-9] объяснялась влиянием электрического поля на вероятность туннелирования дырок из кремниевой подложки в диоксид кремния $\mathrm{SiO}_{2}$. Отметим однако, что преодоление высокого потенциального барьера на МФГ $\mathrm{Si}-\mathrm{SiO}_{2}(\sim 4.6$ эВ $)$ возможно только горячими дырками, которые практически отсутствуют при низких $(E<6 \mathrm{MB} / \mathrm{cm})$ напряженностях электрического поля в $\mathrm{SiO}_{2}$. Полная физическая картина ОТПН продолжает оставаться дискуссионной [12]. 
Депассивация водородных связей на МФГ $\mathrm{Si}-\mathrm{SiO}_{2}$ при ОТПН (так же как и при ионизирующем облучении [13]) может осуществляться по энергетически более выгодной реакции - положительными ионами водорода (протонами) с энергией активации 0.95 эВ вместо 2.1 эВ в реакции (1) [14]:

$$
\mathrm{SiH}+\mathrm{H}^{+} \leftrightarrow \mathrm{Si}^{+} \bullet+\mathrm{H}_{2},
$$

где $\mathrm{SiH}$ - водородосодержащая ловушка на МФГ $\mathrm{Si}-\mathrm{SiO}_{2}$. При этом источником свободного водорода может являться не МФГ $\mathrm{Si}-\mathrm{SiO}_{2}$, как это полагается в Р-Д моделях [1-9], а кремниевая подложка, в которой водород находится как в свободном состоянии, так и связанным в комплексы с легирующими примесями.

В настоящей работе предложена новая количественная модель ОТПН на основе реакции депассивации водородных связей типа (2) положительными ионами водорода, поступающими из объема кремниевой подложки.

\section{2. Описание модели}

После операций термического окисления, формирования подзатворного диэлектрика, затвора и металлизации структура МОП-транзистора оказывается насыщенной водородом как в свободном, так и в связанном состояниях. Концентрация водорода определяется технологической предысторией МОП-структуры и может достигать $10^{20} \mathrm{~cm}^{-3}[10]$. При температуре ОТПН в кремниевой подложке МОП-транзисторов водород становится подвижным и устанавливается равновесие между водородосодержащими компонентами - нейтральными, отрицательно и положительно заряженными атомами водорода $\left(\mathrm{H}^{0}, \mathrm{H}^{-}\right.$и $\left.\mathrm{H}^{+}\right)$:

$$
\begin{aligned}
& \mathrm{H}^{0}+e^{-} \underset{k_{2}}{\stackrel{k_{1}}{\leftrightarrows}} \mathrm{H}^{-}, \\
& \mathrm{H}^{0}+h^{+} \underset{k_{4}}{\stackrel{k_{3}}{\leftrightarrows}} \mathrm{H}^{+},
\end{aligned}
$$

a также комплексами водорода с легирующими примесями, в частности с донорной примесью фосфора в $n$-кремниевой подложке $p$-МОП-транзисторов:

$$
\mathrm{H}^{-}+\mathrm{P}^{+} \underset{k_{6}}{\stackrel{k_{5}}{\leftrightarrows}} \mathrm{P}^{+} \mathrm{H}^{-}
$$

При приложении отрицательного напряжения к затвору $p$-МОП-транзистора выше порогового в приповерхностной области $n$-кремниевой подложки образуется инверсный $p^{+}$-слой, в котором в соответствии c реакцией (4) происходит накопление положительно заряженных ионов водорода. Эти ионы водорода могут переходить из кремния в $\mathrm{SiO}_{2}$ и обратно через МФГ $\mathrm{Si}-\mathrm{SiO}_{2}$, преодолевая относительно невысокий энергетический барьер [15]. Коэффициент сегрегации ионов $\mathrm{H}^{+}$на МФГ $\mathrm{Si}-\mathrm{SiO}_{2}$ должен зависеть от напряженности и полярности электрического поля посредством их влияния на эффективную высоту барьера. После проникновения в $\mathrm{SiO}_{2}$ ионы водорода $\mathrm{H}^{+}$взаимодействуют с пассивированными $P_{b} \mathrm{H}$-центрами и депассивируют их с образованием поверхностных состояний $\left(P_{b}\right)$ и молекулярного водорода по реакции $[14,16]$

$$
P_{b} \mathrm{H}^{0}+\mathrm{H}^{+} \underset{k_{8}}{\stackrel{k_{7}}{\leftrightarrows}} P_{b}^{+}+\mathrm{H}_{2}
$$

В реакции (6) учтено, что поверхностные состояния на МФГ $p^{+}$-инверсного слоя $\mathrm{Si}$ с $\mathrm{SiO}_{2}$ заряжаются положительно. Ионы $\mathrm{H}^{+}$взаимодействуют также с водородосодержащими ловушками $T \mathrm{H}^{0}$, расположенными в $\mathrm{SiO}_{2}$ вблизи МФГ $\mathrm{Si}-\mathrm{SiO}_{2}$, по реакции, аналогичной (6), образуя положительный объемный заряд в диэлектрике:

$$
T \mathrm{H}^{0}+\mathrm{H}^{+} \underset{k_{10}}{\stackrel{k_{9}}{\leftrightarrows}} T^{+}+\mathrm{H}_{2}
$$

Ловушками являются, по-видимому, кислородные вакансии ( $E^{\prime}$-центры), располагающиеся в тонком слое термического $\mathrm{SiO}_{2}$ толщиной $\sim 1-10$ нм, прилегающем к МФГ $\mathrm{Si}-\mathrm{SiO}_{2}[13,17]$. Молекулярный водород, образующийся в реакциях (6) и (7), диффундирует через $\mathrm{SiO}_{2}$ к затвору (обычно сильно легированный поликремний), в котором накапливается, растворяясь или захватываясь на межзеренные границы. При снятии напряжения с затвора накопленный водород может диффундировать обратно из затвора в подзатворный диэлектрик, пассивируя дырочные ловушки вблизи МФГ $\mathrm{Si}-\mathrm{SiO}_{2}$ и поверхностные состояния на $\mathrm{MФГ} \mathrm{Si}-\mathrm{SiO}_{2}$ по обратным реакциям (6) и (7). Другим механизмом релаксации при снятии или изменении напряжения на затворе является туннельная разрядка объемного заряда диэлектрика электронами подложки [18].

Процесс перераспределения и накопления зарядов в МОП структуре в соответствии с реакциями (3)-(7) описываем следующими диффузионно-кинетическими уравнениями непрерывности и уравнением Пуассона:

а) в $\mathrm{Si}\left(\right.$ при $\left.0 \leq x \leq d_{\mathrm{Si}}\right)$

$$
\begin{gathered}
\frac{\partial n}{\partial t}=D_{n} \frac{\partial^{2} n}{\partial x^{2}}+\mu_{n} \frac{\partial}{\partial x}(n E)-k_{1} C_{H}^{0} n+k_{2} C_{H}^{-} \\
\frac{\partial p}{\partial t}=D_{p} \frac{\partial^{2} p}{\partial x^{2}}-\mu_{p} \frac{\partial}{\partial x}(p E)-k_{3} C_{H}^{0} p+k_{4} C_{H}^{+} \\
\frac{\partial C_{H}^{0}}{\partial t}=D_{H}^{0} \frac{\partial^{2} C_{H}^{0}}{\partial x^{2}}-k_{1} C_{H}^{0} n-k_{3} C_{H}^{0} p+k_{2} C_{H}^{-}+k_{4} C_{H}^{+} \\
\frac{\partial C_{H}^{-}}{\partial t}=D_{H}^{-} \frac{\partial^{2} C_{H}^{-}}{\partial x^{2}}+\mu_{H}^{-} \frac{\partial}{\partial x}\left(C_{H}^{-} E\right) \\
\quad+k_{1} C_{H}^{0} n-k_{2} C_{H}^{-}-k_{5} C_{H}^{-} C_{P}^{+}+k_{6} C_{P H}^{0} \\
\frac{\partial C_{H}^{+}}{\partial t}=D_{H}^{+} \frac{\partial^{2} C_{H}^{+}}{\partial x^{2}}-\mu_{H}^{+} \frac{\partial}{\partial x}\left(C_{H}^{+} E\right)+k_{3} C_{H}^{0} p-k_{4} C_{H}^{+} \\
\frac{\partial C_{P H}^{0}}{\partial t}=k_{5} C_{H}^{-} C_{P}^{+}-k_{6} C_{P H}^{0}
\end{gathered}
$$


б) на МФГ $\mathrm{Si}-\mathrm{SiO}_{2}\left(\right.$ при $\left.x=d_{\mathrm{Si}}\right)$

$$
\frac{\partial N_{i t}}{\partial t}=k_{7}\left(N_{i 0}-N_{i t}\right) C_{H}^{+}-k_{8} N_{i t} C_{H 2} ;
$$

в) в объеме $\mathrm{SiO}_{2}$ (при $\left.d_{\mathrm{Si}} \leq x \leq d\right)$

$$
\begin{gathered}
\frac{\partial C_{H}^{+}}{\partial t}=D_{H}^{*} \frac{\partial^{2} C_{H}^{+}}{\partial x^{2}}-\mu_{H}^{*} \frac{\partial}{\partial x}\left(C_{H}^{+} E\right) \\
-k_{9} C_{T H}^{0} C_{H}^{+}+k_{10} C_{T}^{+} C_{H 2}, \\
\frac{\partial C_{P}^{+}}{\partial t}=k_{9} C_{T H}^{0} C_{H}^{+}-k_{10} C_{T}^{+} C_{H 2}-R C_{T}^{+}, \\
\frac{\partial C_{H 2}}{\partial t}=D_{H 2} \frac{\partial^{2} C_{H 2}}{\partial x^{2}}
\end{gathered}
$$

г) в затворе (при $\left.d \leq x \leq d_{G}\right)$

$$
\frac{\partial C_{H 2}}{\partial t}=D_{H 2}^{*} \frac{\partial^{2} C_{H 2}}{\partial x^{2}}
$$

д) во всей МОП-структуре под затвором (при $0 \leq x \leq d)$

$$
\frac{\partial^{2} V}{\partial x^{2}}=-\frac{\rho}{\varepsilon \varepsilon_{0}}
$$

где $x$ - координата, отсчитываемая от нижней границы кремниевой подложки при $x=0$ до границы с подзатворным диэлектриком при $x=d_{\mathrm{Si}}$, далее до границы с затвором при $x=d\left(d=d_{\mathrm{Si}}+d_{o x}\right.$, где $d_{o x}-$ толщина подзатворного диэлектрика) и до верхней границы затвора при $x=d_{G} ; t-$ время; $n$ и $p-$ концентрации свободных электронов и дырок в $\mathrm{Si}$ соответственно; $D_{n}$, $D_{p}, \mu_{n}$ и $\mu_{p}-$ коэффициенты диффузии и подвижности электронов и дырок в $\mathrm{Si} ; C_{H}^{0}, C_{H}^{-}$и $C_{H}^{+}-$концентрации нейтральных атомов, отрицательно и положительно заряженных ионов водорода соответственно; $D_{H}^{0}, D_{H}^{-}, D_{H}^{+}$, $\mu_{H}^{-}$и $\mu_{H}^{+}-$их коэффициенты диффузии и подвижности; $N_{D}$ - концентрация доноров (фосфора) в кремниевой подложке; $C_{P}^{+}$и $C_{P H}^{0}-$ концентрации ионизованных атомов фосфора и их комплексов с водородом в $\mathrm{Si}$ $\left(C_{P}^{+}=N_{D}-C_{P H}^{0}\right) ; N_{i 0}$ и $N_{i t}-$ начальные плотности пассивированных $P_{b} \mathrm{H}$-центров и текущие плотности депассивированных $P_{b}$-центров; $C_{T H}^{0}$ и $C_{T}^{+}$- концентрации нейтральных водородосодержащих и положительно заряженных безводородных ловушек; $D_{H}^{*}$ и $\mu_{H}^{*}-$ коэффициент диффузии и подвижность ионов $\mathrm{H}^{+}$в $\mathrm{SiO}_{2}$; $D_{H 2}$ и $D_{H 2}^{*}-$ коэффициенты диффузии $\mathrm{H}_{2}$ в $\mathrm{SiO}_{2}$ и в затворе соответственно; $V-$ распределение электрического потенциала; $E-$ напряженность электрического поля, $E=-d V / d x ; \rho-$ плотность объемного заряда, $\rho=q\left(p-n+C_{P}^{+}+C_{H}^{+}-C_{H}^{-}+C_{T}^{+}\right), q-$ заряд электрона; $\varepsilon-$ относительная диэлектрическая проницаемость $\mathrm{Si}(\varepsilon(\mathrm{Si})=11.8)$ или $\mathrm{SiO}_{2}\left(\varepsilon\left(\mathrm{SiO}_{2}\right)=3.9\right)$; $\varepsilon_{o}$ - диэлектрическая постоянная; $R$ - вероятность туннельной разрядки положительно заряженных ловушек $T^{+}$, зависящая от распределения ловушек в оксиде и напряженности электрического поля $[18,19]$ :

$$
R=\alpha \exp \left[-\beta(E)\left(x-d_{\mathrm{Si}}\right)\right]
$$

где $\alpha-$ частотный фактор, $\beta-$ барьерный фактор, $\beta(E)=\beta_{0}(1-\gamma E)^{0.5}, \beta_{0}-$ барьерный фактор при $E=0, \gamma=q x_{0} / E_{T}, E_{T}$ - высота барьера, $x_{0}$ - ширина барьера.

Уравнения (8)-(19) решались при следующих начальных и граничных условиях. Начальные концентрации нейтральных, отрицательно и положительно заряженных атомов водорода, а также комплексов водорода с легирующей примесью полагались однородными по глубине кремния (при $0 \leq x \leq d_{\mathrm{Si}}$ ):

$$
\begin{aligned}
& C_{H}^{0}(x, 0)=C_{H 0}^{0}, \quad C_{H}^{-}(x, 0)=C_{H 0}^{-}, \\
& C_{H}^{+}(x, 0)=C_{H 0}^{+}, \quad C_{P H}^{0}(x, 0)=C_{P H 0}^{0}
\end{aligned}
$$

и находящимися в равновесии друг с другом:

$$
\begin{gathered}
C_{H 0}^{-}=C_{H 0}^{0} n k_{1} / k_{2}, \quad C_{H 0}^{+}=C_{H 0}^{0} p k_{3} / k_{4}, \\
C_{P H 0}^{0}=C_{H 0}^{-} C_{P}^{+} k_{5} / k_{6} .
\end{gathered}
$$

Начальная плотность пассивированных $P_{b}$-центров на МФГ $\mathrm{Si}-\mathrm{SiO}_{2}$

$$
N_{i}(0)=N_{i 0} .
$$

Начальную концентрацию водородосодержащих ловушек полагаем неоднородной по глубине $\mathrm{SiO}_{2}$

$$
C_{T H}^{0}(x)=Q_{T H 0}^{0} \exp \left[-\left(x-d_{\mathrm{Si}}\right) / l_{T}\right] / l_{T},
$$

где $Q_{T H 0}^{0}-$ начальная плотность водородосодержащих ловушек, $l_{T}$ - ширина распределения. Нижняя граница кремниевой подложки полагается отражающей для всех подвижных компонентов $\left(\mathrm{H}^{0}, \mathrm{H}^{-}\right.$и $\left.\mathrm{H}^{+}\right)$при $x=0$ :

$$
j_{H}^{0}=j_{H}^{-}=j_{H}^{+}=0 \text {, }
$$

где

$$
\begin{gathered}
j_{H}^{0}=-D_{H}^{0} \frac{\partial C_{H}^{0}}{\partial x}, \quad j_{H}^{-}=-D_{H}^{-} \frac{\partial C_{H}^{-}}{\partial x}-\mu_{H}^{-} C_{H}^{-} E, \\
j_{H}^{+}=-D_{H}^{+} \frac{\partial C_{H}^{+}}{\partial x}+\mu_{H}^{+} C_{H}^{+} E .
\end{gathered}
$$

На МФГ $\mathrm{Si}-\mathrm{SiO}_{2}$ при $x=d_{\mathrm{Si}}$ ионы $\mathrm{H}^{+}$перераспределяются в соответствии с условием непрерывности потока и коэффициентом сегрегации $m_{s}$ :

$$
m_{s 1}=C_{H}^{+}\left(\mathrm{SiO}_{2}\right) / C_{H}^{+}(\mathrm{Si}) .
$$

Полагаем, что электрическое поле влияет на эффективную высоту барьера для ионов $\mathrm{H}^{+}$на МФГ $\mathrm{Si}-\mathrm{SiO}_{2}$, приводя к зависимости коэффициента сегрегации $m_{s 1}$ от напряженности электрического поля вида

$$
m_{s 1}(E)=m_{s 10} \exp (a E / k T),
$$

где $m_{s 10}$ - коэффициент сегрегации при $E=0, a-$ эффективная ширина барьера, $k$ - постоянная Больцмана, 
$T$ - температура. Полагается, что МФГ $\mathrm{Si}-\mathrm{SiO}_{2}$ при $x=d_{\mathrm{Si}}$ является отражающей для молекул $\mathrm{H}_{2}$ :

$$
j_{H 2}=-D_{H 2} \frac{\partial C_{H 2}}{\partial x}=0
$$

a МФГ $\mathrm{SiO}_{2}$-затвор при $x=d$ - отражающей для ионов $\mathrm{H}^{+}$:

$$
j_{H}^{+}=-D_{H}^{+} \frac{\partial C_{H}^{+}}{\partial x}+\mu_{H}^{+} C_{H}^{+} E=0 .
$$

Затвор полагается стоком и накопителем для молекулярного водорода с коэффициентом сегрегации $m_{s}$ на МФГ с затвором (при $x=d)$ :

$$
m_{s 2}=C_{H 2}(\text { Gate }) / C_{H 2}\left(\mathrm{SiO}_{2}\right) .
$$

При ОТПН на затвор подается отрицательное напряжение $V_{G 1}$, а при релаксации - напряжение $V_{G 2}$

$$
V(d, t)=V_{G 1} \text { или } V_{G 2} \text {. }
$$

Сдвиг порогового напряжения МОП-структуры при ОТПН $\Delta V_{t}$ складывается из поверхностной $\Delta V_{i t}$ и объемной $\Delta V_{o t}$ составляющих:

$$
\Delta V_{t}=\Delta V_{i t}+\Delta V_{o t}=-\left(Q_{i t}+Q_{o t}\right) / C_{o x},
$$

где $C_{o x}$ - удельная емкость диэлектрика, $C_{o x}=\varepsilon \varepsilon_{0} / d$, $Q_{o t}$ - изменение объемного заряда в оксиде при ОТПН,

$$
Q_{o t}=q \int_{d_{\mathrm{Si}}}^{d} C_{T}^{+}\left(1-\left(x-d_{o x}\right) / d_{o x}\right) d x,
$$

$Q_{i t}$ - изменение заряда ПС при ОТПН. Заряд ПС в общем случае зависит от поверхностного потенциала, уровня и типа легирования кремниевой подложки, а также от распределения ПС по энергиям. Полагаем, что при отрицательном напряжении на затворе выше порогового и образовании инверсного $p^{+}$-слоя все образующиеся ПС заряжены положительно, т. е.

$$
Q_{i t}=q N_{i t}
$$

Параметрами модели являются константы скоростей реакций (3)-(7) и коэффициенты сегрегации на обеих МФГ. При диффузионном контроле константы скоростей реакций: $k_{1}=4 \pi R_{1} D_{n}, k_{3}=4 \pi R_{3} D_{p}, k_{5}=4 \pi R_{5} D_{H}^{-}$, $k_{8,10}=4 \pi R_{8,10} D_{H 2}, k_{7,9}=4 \pi R_{7,9} D_{H}^{+}$, где $R-$ радиусы захвата, полагаем $R_{1,3,5,7,9}=1 \AA, R_{8,10}=10 \AA$. Константы скоростей обратных реакций в $\mathrm{Si}$ находим из условия равновесия соответствующих реакций:

$$
\begin{gathered}
k_{2}=k_{1} n_{H}, n_{H}=N_{C} \exp \left(\frac{E_{C}-E_{H}^{-}}{k T}\right) ; \\
k_{4}=k_{3} p_{H}, \quad p_{H}=N_{v} \exp \left(\frac{E_{H}^{+}-E_{v}}{k T}\right) ; \\
k_{6}=v_{P H} \exp \left(\frac{E_{P H}^{0}}{k T}\right) .
\end{gathered}
$$

Значения известных параметров брались из экспериментальных работ. В $\mathrm{Si}: D_{H}^{0}=8.4 \exp \left(-\frac{1.12}{k T}\right), D_{H}^{-}=$ $=1.3 \cdot 10^{-2} \exp \left(-\frac{0.8}{k T}\right), \quad E_{H}^{-}=E_{c}-0.06$ эВ, $\quad E_{H}^{+}=E_{v}$ +0.6 эВ, $v_{P H}=10^{13} \mathrm{c}^{-1}, E_{P H}^{0}=1.21$ эВ [20,21]; на МФГ: $N_{i 0}=1 \cdot 10^{13} \mathrm{~cm}^{-2}[22] ;$ в $\mathrm{SiO}_{2}: D_{H}^{*}=1.0 \exp \left(-\frac{0.76}{k T}\right)[23]$, $D_{H 2}=7.2 \cdot 10^{-5} \exp \left(-\frac{0.58}{k T}\right)$ [24]. Полагали на МФГ $\mathrm{SiO}_{2}$-затвор $m_{s 2}=100$, а в затворе $D_{H 2}^{*}=D_{H 2}$. Влияние напряженности электрического поля осуществляется через коэффициент сегрегации ионов $\mathrm{H}^{+}$на МФГ $\mathrm{Si}-\mathrm{SiO}_{2}-m_{s 1}(E)$, даваемым выражением (26), в котором параметры $m_{s 10}$ и $\alpha$ определялись из соответствия расчетов экспериментальным данным.

\section{3. Расчеты по модели}

Система уравнений модели (7)-(19) с начальными условиями (21) - (24), граничными условиями $(25)-(31)$ с учетом (20), (32)-(34) решалась численно с использованием неявной разностной схемы. На рис. 1 показаны распределения дырок (1), электронов (2), ионов водорода $\mathrm{H}^{+}$в кремнии $\left(3-3^{\prime \prime}\right)$ и в $\mathrm{SiO}_{2}\left(4-4^{\prime \prime}\right)$, а также молекулярного водорода $\mathrm{H}_{2}$ в $\mathrm{SiO}_{2}\left(5-5^{\prime \prime}\right)$ и в затворе $\left(6-6^{\prime \prime}\right)$ по глубине МОП-структуры после воздействия ОТПН при $V_{G}=-40$ В в течение $10,10^{3}$ и $10^{5}$ c. При подаче напряжения на затвор больше порогового на поверхности кремния образуется инверсный $p^{+}$-слой, в котором, согласно реакции (4), увеличивается концентрация положительно заряженных ионов водорода $\mathrm{H}^{+}$. В соответствии с коэффициентом сегрегации, зависящим от электрического поля, часть ионов $\mathrm{H}^{+}$переходит в $\mathrm{SiO}_{2}$, где захватывается на МФГ $\mathrm{Si}-\mathrm{SiO}_{2}$ и на водородосодержащие ловушки. В результате происходят реакции депассивации (6) и (7) и об-

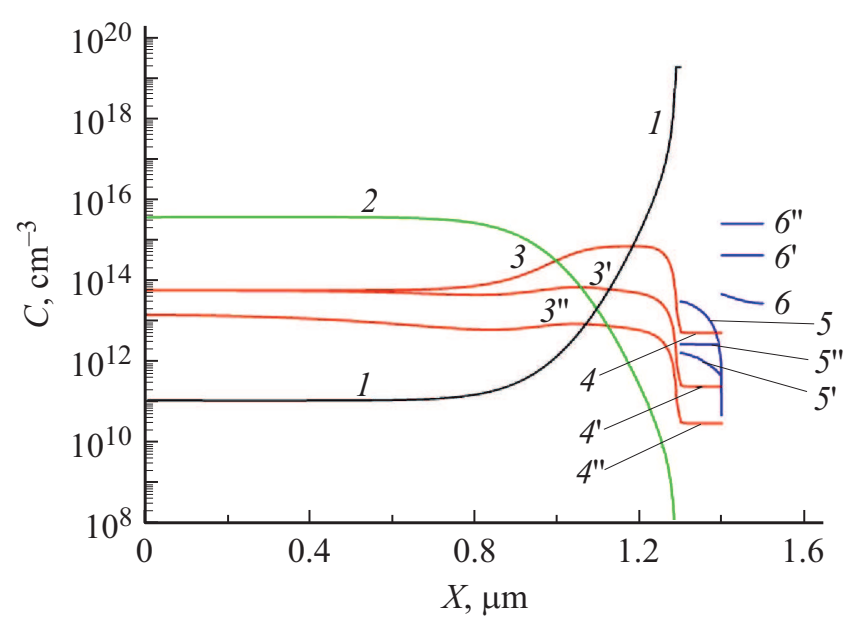

Рис. 1. Расчетные распределения компонентов по глубине МОП-структуры: $1-p ; 2-n ; 3,3^{\prime}, 3^{\prime \prime}-\mathrm{H}^{+}$в $\mathrm{Si}$; $4,4^{\prime}, 4^{\prime \prime}-\mathrm{H}^{+}$в $\mathrm{SiO}_{2} ; 5,5^{\prime}, 5^{\prime \prime}-\mathrm{H}_{2}$ в $\mathrm{Si} ; 6,6^{\prime}, 6^{\prime \prime}-\mathrm{H}_{2}$ в затворе при временах ОТПН, $c: 3-5-10,3^{\prime}-5^{\prime}-10^{3}$, $3^{\prime \prime}-5^{\prime \prime}-10^{5} .\left(V_{G}=-40 \mathrm{~B}, T=150^{\circ} \mathrm{C}, C_{H 0}^{0}=7 \cdot 10^{13} \mathrm{~cm}^{-3}\right.$, $\left.N_{t 0}=3 \cdot 10^{13} \mathrm{~cm}^{-2}, N_{D}=2 \cdot 10^{15} \mathrm{~cm}^{-3}, d_{\mathrm{Si}}=1.3 \mathrm{MKM}\right)$. 


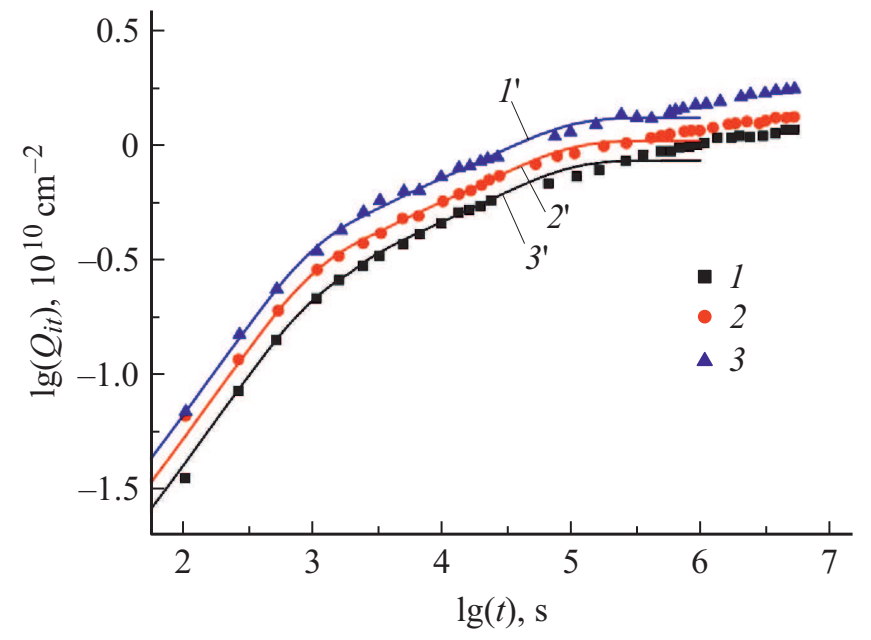

Рис. 2. Зависимости $\Delta Q_{i t}$ от времени ОТПН при $V_{G}$, В: $1,1^{\prime}-$ $35 ; 2,2^{\prime}-40 ; 3,3^{\prime}-45 ; 1-3-$ эксперимент [25], $1^{\prime}-3^{\prime}-$ расчет при значениях: $C_{H 0}^{0}=7 \cdot 10^{13} \mathrm{~cm}^{-3}, N_{t 0}=3 \cdot 10^{13} \mathrm{~cm}^{-2}$, $N_{D}=2 \cdot 10^{15} \mathrm{~cm}^{-3}, d_{\mathrm{Si}}=1.3$ мкм.

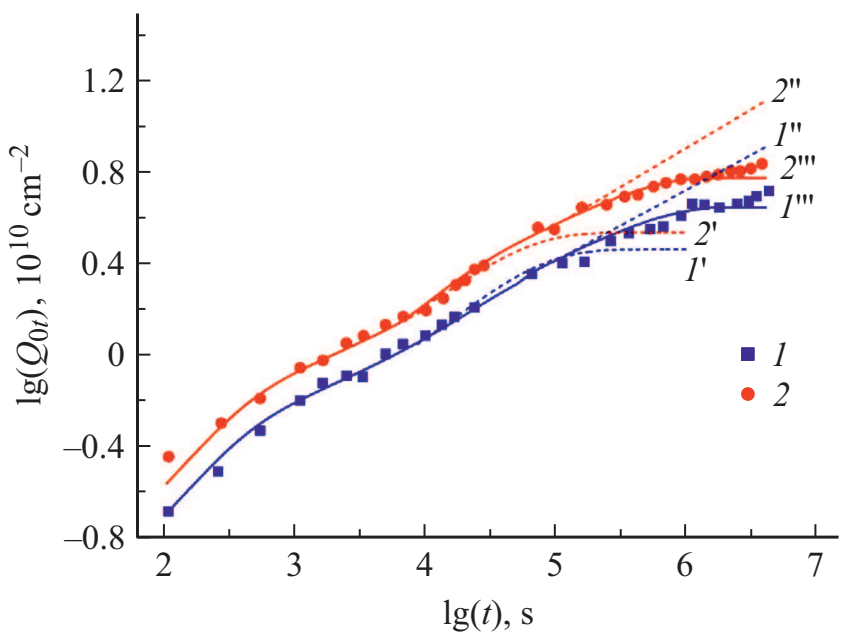

Рис. 3. Зависимости $\Delta Q_{0 t}$ от времени ОТПН при $V_{G}$, B: $1,1^{\prime}, 1^{\prime \prime}, 1^{\prime \prime \prime}-35 ; 2,2^{\prime}, 2^{\prime \prime}, 2^{\prime \prime \prime}-45 ; 1,2$ - эксперимент [25], $1^{\prime}, 1^{\prime \prime}, 1^{\prime \prime \prime}, 2^{\prime}, 2^{\prime \prime}, 2^{\prime \prime \prime}$ - расчет при значениях $d_{\mathrm{Si}}$, мкм: $1^{\prime}, 1^{\prime \prime \prime}, 2^{\prime}, 2^{\prime \prime \prime}-1.3 ; 1^{\prime \prime}, 2^{\prime \prime}-10 ; 1^{\prime \prime \prime}, 2^{\prime \prime \prime}-$ при наличии слоя с бором $\left(N_{\mathrm{B}}=8 \cdot 10^{12} \mathrm{~cm}^{-3}, d_{\mathrm{B}}=0.1 \mathrm{M \kappa м}\right) .\left(C_{H 0}^{0}=7 \cdot 10^{13} \mathrm{~cm}^{-3}\right.$, $\left.N_{t 0}=5 \cdot 10^{13} \mathrm{~cm}^{-2}, N_{D}=1 \cdot 10^{16} \mathrm{~cm}^{-3}\right)$.

разуется молекулярный водород, который диффундирует через объем $\mathrm{SiO}_{2}$ к затвору. Со временем концентрации всех водородосодержащих компонентов в кремниевой подложке убывают вследствие захвата ионов $\mathrm{H}^{+}$ловушками в $\mathrm{SiO}_{2}$ и стока молекулярного водорода в затвор.

Расчеты по модели сравнивались с экспериментальными зависимостями $Q_{i t}(t)$ и $Q_{0 t}(t)$ из работы [25] (значки на рис. 2 и 3), полученными на мощных -канальных VDMOSFET транзисторах типа IRF9520 с $d_{o x}=100 \mathrm{HM}$, после воздействия напряжения на затворе $V_{G}=-35,-40$ и -45 В при температуре $T=150^{\circ} \mathrm{C}$.
Как видно из рис. 2, на зависимостях $\log Q_{i t}$ от $\log t$ имеются три участка. Начальный участок при временах $t=10^{2}-10^{3}$ с имеет показатель степенной зависимости, изменяющийся от $n \cong 1$ до $n \cong 0.5$. На среднем участке при временах $10^{3}<t<10^{5}$ с показатель падает до $n=0.2-0.25$. На конечном третьем участке при больших временах $t>10^{5} \mathrm{c}$ показатель падает до $n=0.1-0.12$. Удовлетворительное соответствие расчетных зависимостей $Q_{i t}(t)$ (кривые $\left.1^{\prime}-3^{\prime}\right)$ с экспериментальными получено на участках 1 и 2 при значениях параметров: $C_{H 0}^{0}=7 \cdot 10^{13} \mathrm{~cm}^{-3}$, $d_{\mathrm{Si}}=1.3$ мкм и $D_{H}^{+}=4.3 \cdot 10^{-13} \mathrm{~cm}^{2} / \mathrm{c}$. Отметим, что найденный коэффициент диффузии ионов водорода в кремнии $D_{H}^{+}$лежит в диапазоне литературных экспериментальных данных [20,26,27]. На участке 3 вместо продолжающегося роста расчет дает насыщение, обусловленное истощением водорода в кремниевой подложке.

Экспериментальные зависимости $Q_{0 t}(t)$ на рис. 3, (значки 1,2 ) на среднем участке с $n=0.25-0.3$ подобны зависимостям $Q_{i t}(t)$ на рис. 2, (значки $\left.1-3\right)$. Начальный участок 1 выражен слабее $(n=0.4-0.5)$, а рост на участке 3 более сильный $(n=0.15-0.16)$. Отличие в поведении заряда ПС $Q_{i t}(t)$ и объемного заряда $Q_{0 t}(t)$ может быть связано с латеральным разделением этих зарядов по площади затвора МОП-структуры. Начальный участок с $n=0.5-1$ сокращается при увеличении концентрации донорной примеси в подложке (кривые $1^{\prime}, 2^{\prime}$ ), что имеет место, например, при заходе затвора на противоинверсную $n^{+}$-область $p$-МОП-транзистора. При больших временах ОТПН так же, как и для $Q_{i t}(t)$ на pис. 2, имеем насыщение расчетной зависимости $Q_{0 t}(t)$ (кривые $\left.1^{\prime}, 2^{\prime}\right)$. Рост $Q_{0 t}(t)$ на участке 3 можно получить при увеличении толщины кремниевой подложки до 10 мкм (кривые $\left.1^{\prime \prime}, 2^{\prime \prime}\right)$, или при введении в нижнюю часть кремниевой подложки слоя, легированного бором, с концентрацией $N_{B}=8 \cdot 10^{12} \mathrm{~cm}^{-3}$ при толщине $d_{B}=0.1$ мкм (кривые $1^{\prime \prime \prime}, 2^{\prime \prime \prime}$ ) с параметрами образования комплексов ВН из работы $[20]\left(v_{B H}=3 \cdot 10^{13} \mathrm{c}^{-1}\right.$, $E_{B H}^{0}=1.28$ эВ).

Влияние напряжения затвора осуществляется в модели посредством зависимости коэффициента сегрегации $m_{s 1}$ от напряженности электрического поля согласно выражению (27). На рис. 2, 3 показаны экспериментальные зависимости $Q_{i t}$ и $Q_{0 t}$ от времени ОТПН из работы [25] при напряжениях на затворе $V_{G}=-35,-40$ и $-45 \mathrm{~B}$ (значки). Соответствие расчетов (кривые $1^{\prime}-3^{\prime}$ на рис. 2 и кривые $1^{\prime \prime \prime}, 2^{\prime \prime \prime}$ на рис. 3) с экспериментальными зависимостями достигалось при следующих значениях параметров в выражении (27): $m_{s 10}=0.04$, $a=2.9 \cdot 10^{-8} \mathrm{~cm}$.

При снятии или изменении смещения затвора происходит уменьшение (релаксация) положительного объемного заряда, накопленного в $\mathrm{SiO}_{2}$ при ОТПН. Полагаем, что процесс релаксации происходит путем туннельной разрядки положительно заряженных оксидных 


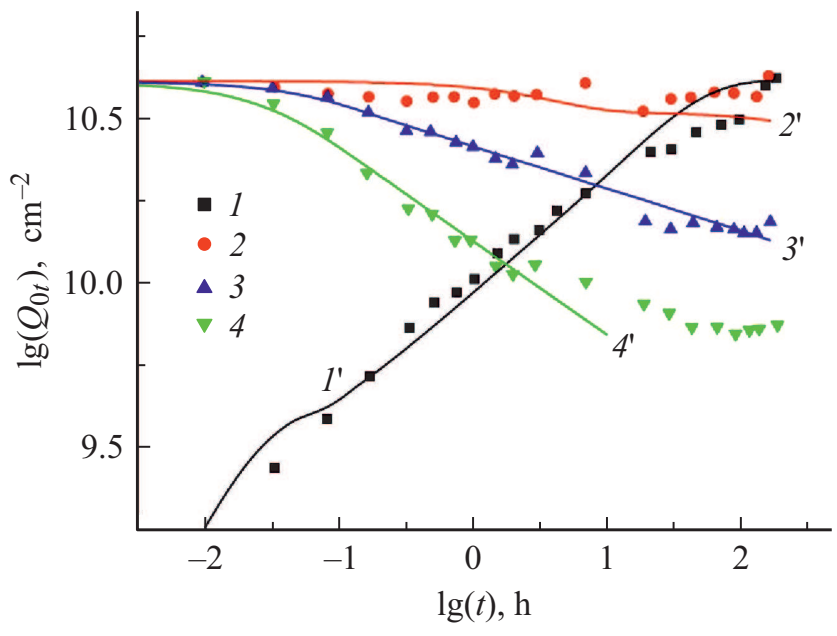

Рис. 4. Зависимости $\Delta Q_{0 t}$ от времени ОТПН при $V_{G 1}=-40 \mathrm{~B}\left(1,1^{\prime}\right)$ и времени релаксации $\left(2,2^{\prime}, 3,3^{\prime}, 4,4^{\prime}\right)$ при $V_{G 2}$, В: 2, $2^{\prime}--10 ; 3,3^{\prime}-0 ; 4,4^{\prime}-+10.1-4-$ эксперимент [28], $1^{\prime}-4^{\prime}-$ расчет при значениях: $C_{H 0}^{0}=6.5 \cdot 10^{13} \mathrm{~cm}^{-3}$, $N_{t 0}=2.9 \cdot 10^{13} \mathrm{~cm}^{-2}, N_{D}=3 \cdot 10^{15} \mathrm{~cm}^{-3}, d_{\mathrm{Si}}=1.9$ мкм.

ловушек электронами из кремниевой подложки. Скорость туннельной разрядки определяется в этом случае выражением (20). Расчеты по модели сравнивались с экспериментальными данными, полученными в работе [28], на $p$-канальных VDMOSFET транзисторах типа IRF9520 с $d_{o x}=100$ нм после воздействия напряжения на затворе $V_{G 1}=-40$ В с последующей выдержкой при напряжениях $V_{G 2}=-10,0$ и $+10 \mathrm{~B}$ при температуре $150^{\circ} \mathrm{C}$. Соответствие расчетов (кривые $1^{\prime}-4^{\prime}$ ) экспериментальным данным (значки $1-4$ на рис. 4) достигалось при следующих значениях параметров в выражении (20): $\alpha=1.5 \cdot 10^{-2} \mathrm{c}^{-1}, \beta_{0}=7.9 \cdot 10^{6} \mathrm{~cm}^{-1}$, $\gamma=8 \cdot 10^{-7} \mathrm{cM} / \mathrm{B}$, что при $x_{0} \cong l_{T}$ соответствует высоте барьера $E_{T} \cong 1,2$ эВ.

\section{4. Обсуждение}

В аналитических решениях Р-Д моделей [1-9] разные показатели связывались с разными состояниями водорода в $\mathrm{SiO}_{2}: n=1 / 6-$ с $\mathrm{H}_{2}, n=1 / 4-\mathrm{c} \mathrm{H}_{0}$; $n=1 / 2-$ с $\mathrm{H}^{+}$. В настоящей модели показатель $n$ на начальном участке $Q_{i t}(t)$ определяется переходом от кинетики химической реакции первого порядка (6) с $n=1$ к кинетике диффузии и дрейфа с $n=0.5$ ионов $\mathrm{H}^{+}$из $p^{+}$-инверсного слоя кремниевой подложки к МФГ $\mathrm{Si}-\mathrm{SiO}_{2}$ сначала без падения их концентрации на нижней границе кремниевой подложки (рис. 1, кривые $3,3^{\prime}$ ). Падение показателя на среднем участке связано с обеднением источника водорода, выражающимся в падении концентрации $\mathrm{H}^{+}$на нижней границе кремниевой подложки при $x=0$ (рис. 1, кривая $3^{\prime \prime}$ ). Одновременно с $\mathrm{H}^{+}$падают концентрации и остальных водородосодержащих компонентов, находящихся в равновесии с $\mathrm{H}^{+}$, что замедляет падение скорости ро- ста $Q_{i t}(t)$ и $Q_{0 t}(t)$ за счет подпитки из этих источников. Энергию активации на этом участке можно оценить как: $E_{a} \cong n E_{D H}^{+} \cong(0.12-0.18)$ эВ, где $E_{D H}^{+}-$энергия активации диффузии $\mathrm{H}^{+}$в кремнии (0.6 эВ [20]), что примерно соответствует экспериментальным значениям 0.15-0.24 эВ в работе [25] и литературным данным $0.1-0.3$ эВ [1-9]. На конечном участке при больших временах ОТПН $\left(t>10^{5} \mathrm{c}\right)$ показатель степенной зависимости показывает дальнейшее падение, что в рамках модели объясняется постепенным истощением источников водорода в кремниевой подложке. При полном истощении водородосодержащих компонентов в кремниевой подложке водород перестает поступать в диэлектрик и показатель на расчетных кривых падает до $n \cong 0$ (насыщение). Экспериментальные зависимости $Q_{i t}(t)$ и $Q_{0 t}(t)$ на рис. 2 и 3 , имея тенденцию к насыщению при больших временах, полного насыщения не достигают. Причиной такого квазинасыщения может быть наличие (помимо рассматриваемого в настоящей одномерной модели планарного источника водорода в кремниевой подложке толщиной $d_{\mathrm{Si}}$ ) дополнительных источников водорода в кремниевой подложке. Такими источниками могут являться, в частности, р-области стока-истока $p$-МОП-транзистора, прилегающие к краям затвора. Действительно, наличие в кремниевой подложке области, легированной бором, с комплексами ВН приводит к дальнейшему росту $Q_{0 t}(t)$ и отодвигает начало насыщения заряда при больших временах ОТПН (кривые $1^{\prime \prime \prime}$ и $2^{\prime \prime \prime}$ на рис. 3). Отметим, что коэффициенты диффузии ионов водорода $\mathrm{H}^{+}$и молекулярного водорода $\mathrm{H}_{2}$ в диоксиде кремния $\left(D_{H}^{*}=8.9 \cdot 10^{-10} \mathrm{~cm}^{2} / \mathrm{c}\right.$, $D_{H 2}=8.9 \cdot 10^{-12} \mathrm{~cm}^{2} /$ с при $150^{\circ} \mathrm{C}$ ) значительно превышают коэффициент диффузии ионов водорода $\mathrm{H}^{+}$в кремнии $\left(D_{H}^{+}=4.3 \cdot 10^{-13} \mathrm{~cm}^{2} / \mathrm{c}\right.$ при $\left.150^{\circ} \mathrm{C}\right)$. Поэтому в настоящей модели, в отличие от Р-Д моделей [1-9], лимитирующей является диффузия водорода в кремнии, а не в $\mathrm{SiO}_{2}$.

Зависимость ОТПН от напряжения на затворе в Р-Д моделях [1-9] объяснялась влиянием электрического поля на вероятность туннелирования дырок из кремниевой подложки в диоксид кремния $\mathrm{SiO}_{2}$. Отметим, что преодоление высокого потенциального барьера $(\sim 4.6$ эВ) возможно только горячими дырками, которые практически отсутствуют при низких напряженностях электрического поля $E<6 \mathrm{MB} / \mathrm{cм}$. В настоящей работе влияние напряжения затвора описывается влиянием электрического поля на коэффициент сегрегации $m_{s}$ ионов $\mathrm{H}^{+}$на МФГ $\mathrm{Si}-\mathrm{SiO}_{2}$. Зависимость $m_{s 1}(E)$ обусловлена влиянием поля на эффективную высоту барьера на $\mathrm{MФГ} \mathrm{Si}-\mathrm{SiO}_{2}$ для ионов $\mathrm{H}^{+}$и имеет экспоненциальный характер от напряженности электрического поля вида (27). Отметим, что эффективная ширина барьера $\left(a=2.9 \cdot 10^{-8}\right.$ см примерно соответствует структурной толщине переходной области $\mathrm{Si}-\mathrm{SiO}_{2}(\sim 0.3 \mathrm{Hм}[29])$.

В Р-Д моделях [1-9] полагалось, что релаксация заряда, накопленного при ОТПН, происходит вследствие уменьшения скорости прямой реакции (1) из-за 
уменьшения концентрации дырок в кремниевой подложке, а также вследствие обратной диффузии водорода в атомном или молекулярном виде от затвора к МФГ $\mathrm{Si}-\mathrm{SiO}_{2}$. Как показано в [2,30], эти процессы не позволяют описать экспериментальные зависимости во всем диапазоне времен релаксации. Кроме того, по обратной реакции (1) можно ожидать релаксацию заряда поверхностных состояний, тогда как в ряде экспериментов $[1,2,28]$ наблюдалась преимущественная релаксация объемного заряда. В настоящей модели процесс релаксации объемного заряда описывается туннельной разрядкой положительно заряженных оксидных ловушек электронами кремниевой подложки. Отметим, что найденные параметры туннелирования $\left(\beta_{0}\right.$ и $\left.E_{T}\right)$ достаточно близки к соответствующим параметрам для туннельной разрядки объемного заряда, введенного ионизирующим облучением, определенным в $[31,32]$.

\section{5. Заключение}

Разработана новая количественная модель нестабильности МОП-приборов при отрицательном смещении затвора. В основе модели лежит реакция депассивации поверхностных состояний на МФГ $\mathrm{Si}-\mathrm{SiO}_{2}$ и водородосодержащих дырочных ловушек в $\mathrm{SiO}_{2}$ положительно заряженными ионами водорода $\mathrm{H}^{+}$, накапливающимися в $p^{+}$-инверсном слое кремниевой подложки. Зависимости поверхностного и объемного зарядов от времени ОТПН определяются кинетикой диффузии и дрейфа ионов $\mathrm{H}^{+}$ из $p^{+}$-инверсного слоя и объема кремниевой подложки к МФГ $\mathrm{Si}-\mathrm{SiO}_{2}$. Модель удовлетворительно описывает экспериментальные зависимости поверхностного и объемного зарядов от времени с показателем степени $n$, изменяющимся от 1 при малых временах до $\sim 0.1$ при больших временах. Квазинасыщение при больших временах связывается с истощением содержания водорода в кремниевой подложке. Влияние напряжения затвора на ОТПН объясняется посредством влияния напряженности электрического поля на коэффициент сегрегации ионов $\mathrm{H}^{+}$на $\mathrm{MФГ} \mathrm{Si}-\mathrm{SiO}_{2}$. Релаксация положительного объемного заряда, введенного в подзатворный диэлектрик при ОТПН, описывается туннельной разрядкой оксидных ловушек электронами кремниевой подложки.

\section{Список литературы}

[1] D.K. Schroder. Microelectron. Reliab., 47, 841 (2007).

[2] A.E. Islam, H. Kufluoglu, D. Varghese, S. Mahapatra, M.A. Alam. IEEE Trans. Electron. Dev., 54 (9), 2143 (2007).

[3] S. Mahapatra, N. Parihar. Microelectron. Reliab., 81, 127 (2018).

[4] V. Huard, M. Denais, C. Parthasarathy. Microelectron. Reliab., 46 (1), 1 (2006).

[5] J.H. Stathis, S. Zafar. Microelectron. Reliab., 46 (2-4), 270 (2006).
[6] K.O. Jeppson, C.M. Swensson. J. Appl. Phys., 48 (5), 2004 (1977).

[7] S. Ogawa, N. Shiono. Phys. Rev. B, 51 (7), 4218 (1995).

[8] J.B. Yang, T.P. Chen, S.S. Tan, L. Chan.. Phys. Lett., 88, 172109 (2006).

[9] H. Kufluoglu, M.A. Alam. IEEE Trans. Electron Dev., 54 (5), 1101 (2007).

[10] D. Griscom. J. Appl. Phys., 58 (7), 2524 (1985).

[11] R.E. Stahlbush, R.K. Lawrence, H.L. Hughes, N.S. Saks. IEEE Trans. Nucl. Sci., 35 (6), 1192 (1988).

[12] J.H. Stathis, S. Mahapatra, T. Grasser. Microelectron. Reliab., 81, 244 (2018).

[13] О.В. Александров. ФТП, 48 (4), 523 (2014).

[14] L. Tsetseris, X.J. Zhou, D.M. Fleetwood, R.D. Schrimpf, S.T. Pantelides. Appl. Phys. Lett., 86, 142103 (2005).

[15] S.T. Pantelides, L. Tsetseris, S.N. Raskeev, X.J. Zhou, D.M. Fleetwood, R.D. Schrimpf. Microelectron. Reliab., 47, 903 (2007).

[16] S.N. Raskeev, D.M. Fleetwood, R.D. Schrimpf, S.T. Pantelides. Phys. Rev. Lett., 87 (16), 165506 (2001).

[17] Q.D.M. Khosru, N. Yasuda, K. Taniguchi, C. Hamaguchi. J. Appl. Phys., 76, 4738 (1994).

[18] P.J. McWhorter, S.L. Miller, W.M. Miller. IEEE Trans. Nucl. Sci., 37 (6), 1682 (1990).

[19] T.R. Oldham, A.J. Lelis, F.B. McLean. IEEE Trans. Nucl. Sci., 33 (6), 1203 (1986).

[20] R. Rizk, P. de Mierry, D. Ballutaud, M. Aucouturier, D. Mathiot. Phys. Rev. B, 44 (12), 6141 (1991).

[21] J. Zhu, N.M. Johnson, C. Herring. Phys. Rev. B, 41 (17), 12354 (1990).

[22] A. Stesmans, G. Van Gorp. Appl. Phys. Lett., 57, 2663 (1990).

[23] S.R. Hofstein. IEEE Trans. Electron Dev., 14 (11), 749 (1967).

[24] B.J. Fishbein, J.T. Watt, J.D. Plummer. J. Electrochem. Soc., 134 (3), 674 (1987).

[25] N. Stojadinovic, D. Dankovic, S. Djoric-Veljkovic, V. Davidovic, I. Manic, S. Golubovic. Microelectron. Reliab., 45, 1343 (2005).

[26] M. Capizzi, A. Mittiga. Appl. Phys. Lett., 50, 918 (1987).

[27] Y.L. Huang, Y. Ma, R. Job, A.G. Ulyashin. J. Appl. Phys., 96, 7080 (2004).

[28] I. Manic, D. Dankovic, S. Djoric-Veljkovic, V. Davidovic, S. Golubovic, N. Stojadinovic. Microelectron. Reliab., 49, 1003 (2009).

[29] Г.Я. Красников, Н.А. Зайцев. Система кремний-диоксид кремния субмикронных СБИС (М., Техносфера, 2003) c. 148 .

[30] K.E. Kambour, C. Kouhestani, D. Nguyen, N. Rosen, R.A.B. Devine. Appl. Phys. Lett., 99, 083506 (2011).

[31] M. Kimura. J. Appl. Phys., 73 (9), 4388 (1993).

[32] J.R. Schwank, P.S. Winokur, P.J. McWhorter, F.W. Sexton, P.V. Dressendorfer, D.C. Turpin. IEEE Trans. Nucl. Sci., 31 (6), 1434 (1984).

Редактор Г.А. Оганесян 


\section{Model of negative bias temperature instability of $p$-MOS-transistors}

\section{O.V. Aleksandrov}

St. Petersburg State Electrotechnical University „LETI“, 197376 St. Petersburg, Russia

Abstract The new quantitative model of negative bias temperature instability (NBTI) of $p$-MOS-transistors is developed. Reaction of depassivation of the surface states on interphase boundary (IFB) $\mathrm{Si}-\mathrm{SiO}_{2}$ and of the hydrogen-related hole traps near IFB $\mathrm{Si}-\mathrm{SiO}_{2}$ by positively charged hydrogen ions $\mathrm{H}^{+}$collecting in $p^{+}$-inverse layer of a silicon substrate is the cornerstone of model. Dependences of surface and volume charges in $p$-MOS-transistors on time of NBTI are defined by kinetics of diffusion and drift of ions $\mathrm{H}^{+}$from a silicon substrate to $\mathrm{IFB} \mathrm{Si}-\mathrm{SiO}_{2}$. Influence of gate voltage on NBTI is explained by means of influence of electric field strength on segregation coefficient of ions $\mathrm{H}^{+}$on IFB $\mathrm{Si}-\mathrm{SiO}_{2}$. The relaxation of the positive volume charge entered into gate dielectric at NBTI is described by a tunnel discharge of oxide traps by electrons of a silicon substrate. 